\title{
New reports of lichenicolous fungi, mainly from Finland and Russia
}

\author{
VAGN ALSTRUP and TEUVO AHTI
}

\begin{abstract}
ALSTRUP, V. \& AHTI, T. 2007: New reports of lichenicolous fungi, mainly from Finland and Russia. - Karstenia 47: 1-4. Helsinki. ISSN 0453-3402.

29 species of lichenicolous fungi are reported from Argentina, Canada, Chile, Finland, India, Mongolia, Russia, and the U.S.A., all presumably new to the country or province of the origin.
\end{abstract}

Vagn Alstrup, Botanical Museum, University of Copenhagen, Gothersgade 130, DK1123 Copenhagen K, Denmark.E-mail:vagna@snm.ku.dk

Teuvo Ahti, Botanical Museum, Finnish Museum of Natural History, P.O. Box 7, FI-00014 Helsinki University, Finland.E-mail:teuvo.ahti@helsinki.fi

The lichenicolous fungi of Finland have been largely neglected until recently. Only a relatively few species are listed in Santesson et al. (2004), but they include a fair number of species originally described from Finland by early authors such as W. Nylander, P. A. Karsten, E. A. Vainio and V. Räsänen. The present list was primarily prepared during the first author's two-week work in the herbarium in Helsinki (H) in August 2006. Apart from collections from Finland and former Finnish parts of Russia, many specimens from other parts of the world are also reported. Several new Finnish records from Peltigera spp. were also discovered but they will be published in forthcoming papers. Only species which are supposed to be new to a country or a province (or other comparable unit) are included. Some specimens could not be identified during the visit and will be treated later on. All the cited collections are preserved in Helsinki $(\mathrm{H})$, if not otherwise mentioned.

The lichenicolous fungi in Russian areas adjacent to Finland in the east have been surveyed rather intensively. A new checklist of Russian lichenicolous fungi prepared by $\mathrm{M}$. Zhurbenko (2007) is in press (the manuscript was available to us). He has recently published several papers on lichenicolous fungi in the Republic of Karelia or Murmansk and Leningrad Regions (e.g.,
Zhurbenko 2001, Zhurbenko \& Himelbrant 2002, Zhurbenko \& Alstrup 2004, Zhurbenko \& Ahti 2005; see also Alstrup et al. 2005), and they also include some records from Finland.

In the list below the nomenclature of the host lichens mainly follows Santesson et al. (2004), if applicable.

Abrothallus bertianus De Not., on Melanelixia and Melanohalea spp.

Finland, Sompion Lappi. Sodankylä, U. K. Kekkonen National Park, Mt. Vongoiva, on Melanohalea olivacea, 1959 Ahti 12976a. - Russia, Pskov Region. Pechory Dist., Verkhulitsa, on M. exasperata, 1991 Ahti et al. 49757a. - Mongolia. Ulaanbaatar, Mt. Bogd Uul, on Melanelixia fuliginosa s. str., 1972 Ahti 29167b. - U.S.A. California. Siskiyou Co., Klamath River town, on Melanohalea multispora, 1984 Ahti \& Norris 42760.

New to Sompion Lappi, Pskov Region, Mongolia, and California.

Abrothallus caerulescens Kotte, on Xanthoparmelia stenophylla (X. somloënsis).

Finland, Varsinais-Suomi. Korpo, Brunskär archipelago, Käringkläppen, 7.VII.1988 Skult; Lohja, Humppila, 3.VI.1892 Boldt. Etelä-Häme. Asikkala, Kalkkinen, Pässivuori, 1986 Ahti 45327.

New to Finland, but earlier cited under $A b$ rothallus parmeliarum. 


\section{Abrothallus cetrariae Kotte, on Platismatia glauca.}

Finland, Varsinais-Suomi. Korpo, Bemby, VIII.1870 Elfving; Sammatti, Kirkonkylä, May 1964, Takala. Pohjois-Savo. Suonenjoki, Kutumäki, 1971 Takala 7507a. Pohjois-Karjala. Lieksa, Egypti, 1875 Vainio; Rautavaara, Pappilanmäki, 1.X.1944 Mannerkorpi; Juuka, Polvela, Tahkovaara, 1971 Takala 7516; Nurmes, Savikylä, 1967 Vitikainen 3606d. - Russia, Leningrad Region, Karelia australis. Gogland, Lounatkorkia, VI.1870 Brenner. - Canada, Newfoundland. Placentia West Dist., Long Pond, 1956 Ahti 6415; St. George's Dist., South Branch Station, 1956 Ahti 7629a. Nova Scotia. Cape Breton Highlands National Park, Lake of Islands, 1965 Scotter $6178 \mathrm{c}$.

New to Finland, Leningrad Region, Newfoundland, and Nova Scotia, but earlier often included in Abrothallus parmeliarum.

\section{Abrothallus parmeliarum (Sommerf. : Fr.) Ar-} nold, on Parmelia spp.

Russia, Sakha Republic. Namskiy Dist., near Arbyn on Lena River, on P. sulcata, 2002 Ahti 61488a. - Mongolia. Ulaanbaatar, Mt. Bogd Uul, on P. saxatilis, 1972 Ahti 29116. - Canada, Newfoundland. Grand Falls Dist., Sandy Lake, on P. saxatilis, 1956 Ahti 8996a.

New to Sakha Republic, Mongolia, and (presumably) Newfoundland.

\section{Abrothallus peyritschii (Stein) Kotte, on Vulpi- cida spp.}

Canada, Northwest Territories. Nahanni National Park, Virginia Falls, on V. tilesii, 1977 Ahti 32481. Ontario. Thunder Bay Dist., Slate Is., Patterson I., on V. pinastri, 1958 Ahti 3976. - India, Himachal Pradesh, Kinnaur Dist., Chitkul, 3900- 4000 m, on V. pinastri, 2003 Upreti et al. 03-002716a.

New to N.W.T., Ontario, and India.

Abrothallus prodiens (Harm.) Diederich \& Hafellner, on Hypogymnia physodes.

Finland, Pohjois-Savo. Suonenjoki, Kutumäki, 1971 Takala $7507 a$.

New to Finland, since the records cited in Santesson et al. (2004) proved to be incorrect or uncertain.

Arthonia peltigerina (Almq.) H. Olivier, on Peltigera patagonica.

Chile, Tierra del Fuego, Isla Grande, Río Caleta, 1986 Stenroos 2259 a (with Illosporium carneum).

New to Chile.

Dactylospora protothallina (Anzi) Hafellner, on Protopannaria pezizoides.

Finland, Koillismaa. Kuusamo, Ylä-Aventojoki, 2.VII.1939 Laurila.

New to Finland.
Echinothecium reticulatum Zopf, on Parmelia saxatilis.

Finland, Etelä-Häme. Hollola, Vaania, Isovuori, 2003 Haikonen 23126; Asikkala: Kirkonseutu, 2003 Haikonen 23148.

New to Finland.

Epicladonia simplex D. Hawksw., on Cladonia cf. merochlorophaea.

Russia, Tuva Republic, Todginskaya Valley, State Reserve Azas, Azas Lake, 18.VIII.1997 Otnyukova.

New to Tuva Republic,

Homostegia piggotii (Berk. \& Broome) P. Karst., on Parmelia spp.

Finland, Keski-Pohjanmaa. Kälviä, Peitso, on $P$. saxatilis, 1966 Takala 136. Inarin Lappi. Utsjoki, Kevo, Kotkapahta, on P. saxatilis, 1965 Ahti 20916a. - Canada, Newfoundland. Ferryland Dist., Tor's Cove WNW, on P. omphalodes s. str., 1956 Ahti 2653. - India, Uttaranchal. Chakrata, on P. sp., 1949 Awasthi 492.

New to the Finnish provinces, Newfoundland, and India.

Karsteniomyces peltigerae (P. Karst.) D. Hawksw., on Peltigera rufescens.

Russia, Sakha Republic. Ust'-Aldanskiy Dist., Aldan River, mouth of Tumara River, 2002 Ahti 61530.

New to Sakha Republic.

Libertiella malmedyensis Speg. \& Roum., on Peltigera rufescens.

USA, New Mexico. Bernalillo Co., Manzano Mts., 4 km NW of Escobosa, 2250- 2300 m, 2001 Ahti $60820 a$.

New to New Mexico.

\section{Lichenoconium cargillianum (Linds.)}

D.

Hawksw., on Melanohalea septentrionalis.

Finland, Koillismaa. Kuusamo, NW shore of Toranginjärvi, 1965 Ahti 19076.

New to Europe.

Lichenoconium lecanorae (Jaap) D. Hawksw., on Lecanora symmicta $\mathrm{s}$. lat.

Finland, Koillismaa. Kuusamo, Lämsänkylä, Sänkelä, 1966 Ahti $22752 c$.

New to Koillismaa.

Lichenoconium usneae (Anzi) D. Hawksw., on Cladonia uncialis.

Finland, Etelä-Häme. Hollola, Vaania, Isovuori, 2003 Haikonen 23137.

New to Finland. 
Lichenostigma arctoparmeliae R. Sant. (ined.), on Arctoparmelia centrifuga.

Finland, Etelä-Savo. Kangasniemi, Puula, Iso Paatsalo, Korppivuori, 2004 Haikonen 23637b. 194).

New to Finland (cf. Santesson et al. 2004:

Lichenostigma cosmopolites Hafellner \& Calatayud, on Xanthoparmelia stenophylla.

Finland, Åland. Jomala, Västansunda, Degerberget, 6.V. 1940 Fagerström. Varsinais-Suomi. Korpo, Brunskär Island, 18.V. 1986 Skult.

New to Finland.

Merismatium nigritellum (Nyl.) Vouaux, on $\mathrm{Ne}$ phroma arcticum.

Finland, Pohjois-Savo. Pieksämäki, Jäppilä, Ruuhilampi, Kontiovuori, 2002 Haikonen 21396.

New to Finland.

Muellerella lichenicola (Sommerf. : Fr.) D. Hawksw., on Caloplaca spp.

Finland, Etelä-Savo. Taipalsaari, Karhunpää, Kallioniemi, on C. holocarpa (C. pyracea), 1991 Vitikainen 12410. Koillismaa. Kuusamo, Virkkula, Mt. Konttainen, on C. borealis, 1980 Ahti 38143.

New to Finland.

Neolamya peltigerae (Mont.) Theiss. \& Syd., on Peltigera rufescens.

Russia, Khabarovsk Territory. Ul'chskiy Dist., Sofiysk, 1976 Alanko 31685.

New to Khabarovsk Territory.

Phacopsis cephalodioides (Nyl.) Triebel \& Rambold, on Hypogymnia vittata.

Finland, Inarin Lappi. Inari, Seävnulvaara (8 km NE of Menesjärvi), 1970 Vitikainen $6406 b$.

New to Finland.

Phacopsis oxyspora (Tul.) A. Massal., on Melanohalea olivacea.

Finland, Inarin Lappi. Utsjoki, Kevo Nature Park, Linkkapahta, 1965 Laine \& Ahti 20961 b.

New to Inarin Lappi.

Phaeopyxis punctum (A. Massal.) Rambold, on Cladonia spp (unidentified squamules).

Finland, Etelä-Häme. Iitti, Sitikkala, Kautionvuori, 1997 Haikonen 18950. Pohjois-Häme. Saarijärvi, Pyhähäkki National Park, 1960 Vitikainen 2182.

New to Etelä-Häme and Pohjois-Häme.
Phaeospora peltigericola D. Hawksw., on Peltigera rufescens.

Canada, Alberta. Peter Lougheed Provincial Park, Kananaskis Lake region, 1700- 2075 m, 1986 Scotter 60778 .

New to Alberta.

Sphaerellothecium minutum Hafellner, on Sphaerophorus globosus.

Argentina, Tierra del Fuego. Depto. Río Grande, $7 \mathrm{~km}$ SE of Estancia Río Apen, 1989 Ahti $47822 a$ (H, UPS).

New to Argentina.

Stigmidium ephebes (Henssen) D. Hawksw., mostly on Ephebe lanata.

Finland, Ảland. Lemland, Lemböte, 1923 Kari. Varsinais-Suomi. Pargas, 1910 Linkola; Naantali, 1937 Häyrén. Uusimaa. Helsinki, 1895 Häyrén; Helsinki, Isosaari, 1927 Linkola. Pohjois-Häme. Jyväskylä, Kanavuori, 1933 Häyrén. Inarin Lappi. Inari, Kaamasjoensuu, on E. hispidula, 1877 Silén. - Russia, Leningrad Region. Karelia australis. Gogland, Maahellinkallio, 1868 Brenner. Karelian Republic, Karelia ladogensis. Kurkijoki, Heposaari, 1932 Räsänen.

New to the Finnish provinces, Leningrad Region and Karelian Republic. It is often overlooked that in the protologue of $S$. ephebes (Henssen 1963) the species was also reported from the Czech Republic (Sudetic Mts.) through citation of Kerner, Fl. Exs. Austro-Hung. No. 3535, though the country is lacking in Henssen's list.

Stigmidium solorinarium (Vain.) D. Hawksw., on Solorina saccata.

Finland, Etelä-Häme. Heinola, Mäyrämäki, 2002 Haikonen 21331. Enontekiön Lappi. Enontekiö, Kilpisjärvi, Saana, 1955 Henssen Le $510 a$.

New to Finland.

Syzygospora bachmannii Diederich \& M. S. Christ., on Cladonia spp.

Finland, Inarin Lappi. Utsjoki, Utsjoki village, on Tana River, on C. gracilis subsp. elongata, 1959 Ahti 12283. - Russia, Leningrad Region, Karelia australis. Vyborg District, Primorsk (Koivisto), 1851, Nylander; Sovetskiy - Landyshevka ("St. Johannes, Kyrkobyn - Kirjola"), 1893 Boldt.

New to Inarin Lappi and Leningrad Region. 
Acknowledgements: The authors are grateful for help by Dr. Orvo Vitikainen, who supplied several collections for this study, and by Dr. Mikhail Zhurbenko who checked whether the Russian collections had been reported before from the provinces mentioned. Prof. Dr. Aino Henssen has identified the specimens of Stigmidium ephebes. The first author's stay in Finland was part of the project "Monitoring lichens - monitoring with lichens" financed by a Nordplus Neighbour grant from the Nordic Council of Ministers.

\section{References}

Alstrup, V., Zavarzin, A. A., Kocourcova, A., Kravchenko, A. V., Fadeeva, M. A. \& Shiefelbein, U. 2005: Lichens and lichenicolous fungi found in Northern Ladoga area (Republic of Karelia) during the international fieldtrip in August 2004, prior to the Fifth Congress of International Lichenological Association. - Trudy Karel'skogo Nauchnogo Tsentra RAN 7: 3-15. (In Russian).

Henssen, A. 1963: Eine Revision der Flechtenfamilien Lichinaceae und Ephebaceae. - Symb. Bot. Ups. 18(1): 1-123.
Santesson, R., Moberg, R., Nordin, A., Tønsberg, T. \& Vitikainen, O. 2004: Lichen-forming and lichenicolous fungi of Fennoscandia. - $359 \mathrm{pp}$. Museum of Evolution, Uppsala University.

Zhurbenko, M. P. 2001: Lichenicolous fungi from Murmansk Region of Russia. - Mikol. Fitopatol. 35: 34-39.

Zhurbenko, M. 2007: Lichenicolous fungi of Russia: annotated list and exploration synthesis. - Mikol. Fitopatol. (in press).

Zhurbenko, M. P. \& Ahti, T. 2005: A preliminary list of lichenicolous fungi of Republic of Karelia, Russia. In: Fungi in natural and anthropogenic ecosystems. Proceedings of international conference dedicated to the centenary of the beginning of professor A. S. Bondartsev his research activity at the V. L. Komarov Botanical Institute RAS 1: 194-200.

Zhurbenko, M. P. \& Alstrup, V. 2004: Lichenicolous fungi on Cladonia mainly from the Arctic. - Symb. Bot. Ups. 34(1): 477-499.

Zhurbenko, M. P. \& Himelbrant, D. E. 2002: Lichenicolous fungi from the Kandalaksha Gulf, Karelia Keretina, Russia. - Folia Cryptog. Estonica 39: 5159. 